\title{
Genomics Approaches for Crop Improvement against Abiotic Stress
}

\author{
Bala Anı Akpınar, ${ }^{1}$ Stuart J. Lucas, ${ }^{1,2}$ and Hikmet Budak ${ }^{1,2}$ \\ ${ }^{1}$ Faculty of Engineering and Natural Sciences, Sabanci University, Orhanl, Tuzla, 34956 Istanbul, Turkey \\ ${ }^{2}$ Sabanci University Nanotechnology Research and Application Centre (SUNUM), Sabanci University, Orhanl, \\ Tuzla, 34956 Istanbul, Turkey \\ Correspondence should be addressed to Hikmet Budak; budak@sabanciuniv.edu
}

Received 4 March 2013; Accepted 22 April 2013

Academic Editors: A. Levine and Z. Wang

Copyright (C) 2013 Bala Anı Akpınar et al. This is an open access article distributed under the Creative Commons Attribution License, which permits unrestricted use, distribution, and reproduction in any medium, provided the original work is properly cited.

\begin{abstract}
As sessile organisms, plants are inevitably exposed to one or a combination of stress factors every now and then throughout their growth and development. Stress responses vary considerably even in the same plant species; stress-susceptible genotypes are at one extreme, and stress-tolerant ones are at the other. Elucidation of the stress responses of crop plants is of extreme relevance, considering the central role of crops in food and biofuel production. Crop improvement has been a traditional issue to increase yields and enhance stress tolerance; however, crop improvement against abiotic stresses has been particularly compelling, given the complex nature of these stresses. As traditional strategies for crop improvement approach their limits, the era of genomics research has arisen with new and promising perspectives in breeding improved varieties against abiotic stresses.
\end{abstract}

\section{Introduction}

Abiotic stresses are the most significant causes of yield losses in plants, implicated to reduce yields by as much as $50 \%$ [1]. Among abiotic stresses, drought is the most prominent and widespread; consequently the drought stress response has been dissected into its components and extensively studied in order to understand tolerance mechanisms thoroughly [2]. To improve abiotic stress, particularly drought, tolerance of cereals is of extreme importance, as cereals, including wheat and barley, are the main constituents of the world food supply. However, many abiotic stresses are complex in nature, controlled by networks of genetic and environmental factors that hamper breeding strategies [3]. As traditional approaches for crop improvement reach their limits, agriculture has to adopt novel approaches to meet the demands of an evergrowing world population.

Recent technological advances and the aforementioned agricultural challenges have led to the emergence of highthroughput tools to explore and exploit plant genomes for crop improvement. These genomics-based approaches aim to decipher the entire genome, including genic and intergenic regions, to gain insights into plant molecular responses which will in turn provide specific strategies for crop improvement. In this paper, genomics approaches for crop improvement against abiotic stresses will be discussed under three generalized classes, functional, structural, and comparative genomics. However, it should be noted that genomics approaches are highly intermingled, in terms of both the methodologies and the outcome (Figure 1).

\section{Functional Genomics}

Genomics research is frequently realized by functional studies, which produce perhaps the most readily applicable information for crop improvement. Functional genomics techniques have long been adopted to unravel gene functions and the interactions between genes in regulatory networks, which can be exploited to generate improved varieties. Functional genomics approaches predominantly employ sequence or hybridization based methodologies which are discussed below. 


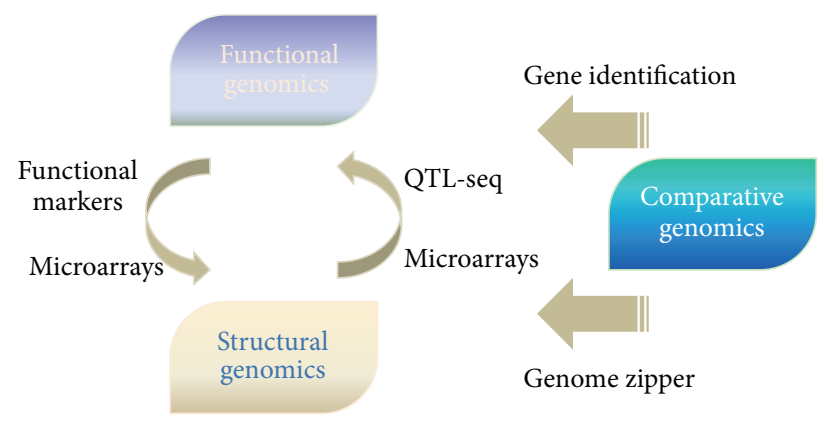

FIgURE 1: Functional, structural, and comparative genomics approaches are highly interrelated. For example, microarrays can be used either to anchor markers to genome maps or to analyze gene expression; functional markers indicate both phenotypes and genetic locations; QTL-seq utilizes a reference genome sequence to isolate QTLs based on phenotypic variation. As more structural genomics information becomes available, comparative genomics tools such as genome zippers can be used both to elucidate the structure of unsequenced genomes and as a shortcut to design targeted functional studies.

2.1. Sequencing-Based Approaches. One way to explore the expressed gene catalogue of a species is to analyze Expressed Sequence Tags (ESTs). ESTs are partial genic sequences that are generated by single-pass sequencing of cDNA clones [4]. Despite the concerns over the quality of ESTs as well as the representation of the parental cDNA [5], ESTs have been shown to identify corresponding genes unambiguously in a rapid and cost-effective fashion [4]; therefore, ESTs have been a major focus on functional studies.

Large-scale EST sequencing has been one of the earliest strategies for gene discovery and genome annotation $[5,6]$. Currently, over a million ESTs are deposited in the EST database at National Center for Biotechnological Information (NCBI) for important crops such as maize, soybean, wheat, and rice, along with several thousands of ESTs for other plants (http://www.ncbi.nlm.nih.gov/dbEST/). cDNA libraries from various tissues, developmental stages, or treatments generally serve as the sources for EST sequencing to reveal differentially expressed genes [7]. These approaches can successfully identify tissue or developmental stage-specific and treatmentresponsive transcripts. However, such cDNA libraries may underrepresent rare transcripts or transcripts that are not expressed under certain conditions. In addition, ESTs are usually much shorter in length than the cDNAs from which they are obtained. Assembly of overlapping EST sequences into consensus contigs is likely to be more informative on the structure of the parental cDNA, which may reveal polymorphisms. However, assembly and interpretation must be handled cautiously, as paralogous genes may lead to misassemblies of sequences, particularly in polyploid species such as wheat [5]. EST sequencing is utilized extensively in the absence of whole genome sequences, particularly in crops with large and repetitive genomes, although the entire transcriptome is unlikely to be fully represented and resolved. Even so, EST sequencing is still a valid approach, and a recent study has demonstrated its potential in gene discovery via the comparison of different genotypes under control and stress conditions [8].

An alternative approach, Serial Analysis of Gene Expression (SAGE), has been developed to quantitate the abundance of thousands of transcripts simultaneously. In this approach, short sequence tags from transcripts are concatenated and sequenced, giving an absolute measure of gene expression $[9,10]$. The ability of these short tags to identify genes unambiguously depends on the existence of comprehensive EST databases for the respective species [11]. Although SAGE is not widely applied in plants, there are a number of examples, including modifications to the original methodology, such as SuperSAGE and DeepSAGE [12-17]. The first report of SAGE in plants not only identified novel genes but also implied novel functions for known genes in rice seedlings [12]. SAGE has also been used to investigate stress-responsive genes $[12,15]$. A similar tag-based approach, Massively Parallel Signature Sequencing (MPSS), where longer sequence tags are ligated to microbeads and sequenced in parallel, enables analysis of millions of transcripts simultaneously [18]. Due to longer tags and high-throughput analysis, MPSS is likely to identify genes with greater specificity and sensitivity. The ability of MPSS to capture rare transcripts is particularly beneficial in species that lack a whole genome sequence [19]. In plants, besides mRNA transcripts, MPSS has been employed in the expression studies of small RNAs [20, 21], which are increasingly implicated in abiotic stress responses [22]. Currently, plant MPSS databases (http://mpss.udel.edu/) contain publicly available MPSS expression data for a number of plant species, including important crops such as rice, maize, and soybean [23]. These MPSS data can be extracted, compiled, and compared with newly generated MPSS data for functional analysis of gene expression, as demonstrated by Jain et al. [24].

2.2. Hybridization-Based Approaches. In contrast to sequence-based approaches, array-based techniques utilize hybridization of the target DNA with cDNA or oligonucleotide probes attached to a surface to assess expression $[25,26]$. These array-based methods are targeted; that is, prior knowledge of the transcript to be analyzed, either sequence or clone, is a prerequisite to design probes [27]. Extensive microarray expression data exists for Arabidopsis thaliana and rice [28-31], model species with fully sequenced genomes. In addition, microarray studies have been widely employed in crop species such as wheat [32], barley [33], and maize [34], as well as less emphasized but still industrially and agriculturally important plant species, such as cotton [35], cassava [36], and tomato [37] to unravel stress responses.

Besides inherent limitations such as cross-hybridization and background noise, microarray studies investigating stress-responsive genes suffer from technical considerations that may limit their usefulness. Isolation of total RNA from complex tissues that are composed of different types of cells may obscure transcript changes occurring in cell types that are particularly relevant to the stress response. Subtle transcriptional changes may be diluted in the overall stress response of the whole tissue and, thus, remain unnoticed. 
Similarly, the choice of tissue or genotype that is sampled in a microarray study is closely related to the relevance of results. Reproductive tissues and stress-tolerant genotypes are most relevant in terms of agricultural gain and stress adaptation mechanisms, respectively [38]. In addition, laboratory-based stress treatments rarely represent field conditions, where multiple stresses usually act together. Interestingly, a comparison of microarray studies carried out using different water deficit stress conditions revealed only a small number of commonly regulated genes [39]. Abiotic stresses are generally complex in nature, eliciting intricate mechanisms of responses in plants. Consequently, slight differences in the experimental application of stress conditions may produce significant differences in stress responses. A further caveat when interpreting microarray studies is that many transcripts are known to undergo posttranscriptional and posttranslational modifications, which results in uncorrelated transcriptomic and proteomic data in some cases.

For species with an available whole genome sequence, a successful expansion of array-based transcript profiling is whole genome tiling arrays [27]. Tiling arrays can identify novel transcriptional units on chromosomes and alternative splice sites and can map transcripts and methylation sites $[40,41]$. Tiling arrays have already been applied in model species to investigate abiotic stress responses [42-44].

\subsection{Expansions to Functional Genomics Approaches. Genome} wide expression profiles are most useful in the detection of candidate genes for desired traits, such as stress tolerance. A fraction of functional studies then adopt inactivation or overexpression of such candidate genes for further characterization and utilization. Of these, Targeting Induced Local Lesions IN Genomes (TILLING) enables high-throughput analysis of large number of mutants [45]. TILLING is applicable to virtually all genes in all species where mutations can be induced and has been reported in several crop species, including hexaploid wheat [46]. TILLING mutants are reported in sorghum [47], maize [48], barley [49], soybean [50], rice [51], and other crops. Although TILLING populations are conventionally screened by phenotypic or genotypic variations, further use of certain TILLING mutants in elucidation of stress responses has been demonstrated. In such a study, TILLING mutants for a specific kinase were used to assess salt stress response in legume species [52].

Importantly, a modified strategy, called EcoTILLING, has been developed to identify natural polymorphisms, analogous to TILLING-assisted identification of induced mutations. Polymorphisms demonstrating natural variation in germplasms are valuable tools in genetic mapping. Furthermore, via the discovery of polymorphisms among individuals, EcoTILLING is able to implicate favorable haplotypes for further analyses, such as sequencing. Similar to TILLING, EcoTILLING is applicable to polyploid species, where it can be utilized to differentiate between alleles of homologous and paralogous genes [53]. In a recent study, EcoTILLING not only provided allelic variants of a number of genes involved in salt stress response but also emphasized the complex nature of salt stress; salt-tolerant genotypes were revealed to harbor different combinations of favorable alleles indicating the presence of multiple pathways conferring salt stress tolerance [54]. Transcription factors, diversifying stress responses, have also been targeted via EcoTILLING to examine natural rice variants exposed to drought stress [55].

The availability of comprehensive EST databases is central to the success of the above-mentioned approaches to identify genes accurately and unambiguously. Besides their utility in genome annotation and expression profiling, ESTs also provide a source of sequences for designing "functional markers." Functional markers refer to polymorphic sites on genes that are attributed to phenotypic variation of traits among individuals of a species. Functional marker design requires the knowledge of the allelic sequences of functionally characterized genes [56]. In contrast to random DNA markers, functional markers are completely linked to the trait of interest; hence, these markers are also called "perfect markers." The use of random DNA markers in breeding studies necessitates validation and revalidation of linkage between the marker and the trait over generations, since genetic recombination may break the linkage $[56,57]$. In addition, functional markers may explore natural variation and biodiversity better, particularly compared to random DNA markers with absence/presence polymorphisms, where allelic variations of a trait exceed that of the linked DNA marker. In the case of such random DNA markers, the locus tested during genotyping will only exhibit biallelic variation, whereas the linked gene may actually have more variants [56]. The importance of functional markers has been highlighted in stress tolerance studies as well $[58,59]$.

\section{Structural Genomics}

While functional genomics focus on the functions of genes and gene networks, structural genomics focus on the physical structure of the genome, aiming to identify, locate, and order genomic features along chromosomes. Together, structural genomics and functional genomics can characterize a genome to its full extent.

3.1. Genome Sequencing and Mapping. In the last decade, advances in DNA sequencing technologies have enabled the generation of a wealth of sequence information including whole genome sequences. Next-generation sequencing (NGS) platforms such as Roche 454 GS FLX Titanium (http://www.454.com/) or Illumina Solexa Genome Analyzer (http://www.illumina.com/) can carry out high capacity sequencing at reduced costs and increased rates compared to conventional Sanger sequencing [60]. These advances have paved the way for the exploitation of plant genomics studies for breeding improved varieties. Through NGS technologies, sequencing and resequencing of even large genomes have become feasible. Accordingly, reference or draft genome sequences for a number of species, including the model species Arabidopsis thaliana and Brachypodium distachyon, along with important crop species such as rice, sorghum, soybean, and maize, have been published [61]. Whole genome sequences provide remarkably detailed information 
on genomic features including coding and noncoding genes, regulatory sequences, repetitive elements, and GC content which can be exploited in functional studies such as microarray or tiling arrays [41]. A high-quality reference genome sequence is considered pivotal to crop improvement via molecular breeding, particularly for complex traits. Despite their usefulness, producing such reference genomes requires a major investment of resources, and currently they are only available for species with relatively small genomes of low repetitive content [61].

Triticeae genomics, including that of the staple crops barley and wheat, has lagged behind recent advances primarily due to their large and complex genomes ( $\sim \mathrm{Gb}$ for barley and $\sim 17 \mathrm{~Gb}$ for wheat) [62]. As pointed out by Morrell et al. [61], 25x coverage sequencing of Drosophila is equivalent to approximately $1 \mathrm{x}$ coverage of wheat genome in terms of sequence read counts, demonstrating the challenging genome size of wheat. The high content of repetitive elements is another major challenge, causing ambiguities in sequence assembly. In polyploid species such as wheat, the sequence assembly problem is further exacerbated due to the presence of homoeologous genomes and paralogous loci [61]. For such genomes, construction of a reference sequence has been considered unattainable until recently.

Over the last few years, advances in chromosome sorting technologies have enabled construction of chromosomespecific Bacterial Artificial Chromosome (BAC) libraries to tackle the challenges of complex genomes. Physical mapping of the $1 \mathrm{~Gb}$ chromosome $3 \mathrm{~B}$ of hexaploid wheat has proven the feasibility of a chromosome-by-chromosome approach to explore and exploit complex genomes [63]. Physical maps not only compile genetic mapping data into physical contigs but also serve as scaffolds for sequence assembly into a reference genome. The physical mapping and reference genome sequencing of wheat and barley are ongoing with combined efforts from a number of consortia [62].

In the absence of reference genome sequences, whole genome or BAC-end shotgun sequences provide valuable insights into genome structure and evolution [64-69]. Intriguingly, whole genome shotgun sequences have also been proposed for Quantitative Trait Loci (QTL) detection via a very recently developed methodology named QTL-seq. In this method, extremes of a population exhibiting a normal distribution with respect to a trait of interest are bulked, sequenced, and compared to detect putative QTLs [70].

3.2. Molecular Markers. Genomics applications involving molecular markers are largely dominated by Single Nucleotide Polymorphisms (SNPs) [71] as reflected in the predominance of software related to SNP discovery [60]. The high abundance of SNPs in genomes is particularly beneficial for their use in genomics. SNPs are readily identified by genome or transcript resequencing and by comparison of different genotypes in species where reference genome sequences or extensive transcript databases are available. Transcriptome resequencing not only avoids repetitive sequences of complex genome but also identifies SNPs within transcripts that may serve as functional markers [72]. However, due to low-quality sequences obtained by most NGS platforms, over-sampling may be required to differentiate SNPs from sequencing errors [71]. In addition, the presence of homoeologous and paralogous loci must be taken into account in SNP identification in polyploid species [72]. Despite the challenges of SNP discovery on the repetitive portion of genomes, efforts are underway to improve SNP identification even in genepoor regions [73]. In fact, these regions are of functional importance as well; for example, an important vernalization gene $V r n-D 4$ has recently been mapped to the centromeric region of chromosome $5 \mathrm{D}$ of hexaploid wheat $[62,74]$.

A recently developed molecular marker type, Insertion Site-Based Polymorphisms (ISBPs), utilizes the insertional polymorphisms observed in the repeat junctions of complex genomes [75]. ISBP markers are readily designed from low coverage shotgun sequences, such as BAC-end sequences $[64,69]$. Typically, 50-60\% of ISBP markers tested are specific for the locus from which they were designed, and in one study which these $\sim 70 \%$ contained SNPs in at least some members of a panel of 14 wheat genotypes [75]. This approach may break the ground for genome saturation particularly for crops with highly repetitive genomes that are impractical to exploit otherwise.

\subsection{Applications of Structural Genomics in Crop Improve-} ment. A major impact of NGS-mediated shotgun sequences has been their substantial contribution to the development of molecular markers. These markers indicate diagnostic polymorphisms at the DNA sequence level, and in contrast to morphological markers which once had been the focus of traditional breeding studies, they are not affected by the environment [76]. In general terms, Marker-Assisted Selection (MAS) refers to the utilization of molecular markers in breeding improved varieties with respect to desired traits, such pathogen resistance, abiotic stress tolerance, or high yield [77]. Through MAS, phenotype can be predicted from genotype [71]. For efficient and accurate MAS, the trait of interest should be tightly linked to a molecular marker [78] or more preferably flanked by two close markers. Recombination between both flanking markers and the trait is less likely to occur compared to a single marker, due to the low frequency of double crossovers. In both cases, a genetic distance of less than $5 \mathrm{cM}$ for each marker from the trait is crucial to the success of MAS [77].

Additionally, for efficient MAS, markers should be highly polymorphic in the germplasm used for breeding. MAS can make use of molecular markers at multiple levels. Plant breeding depends on genetic diversity to improve crops [57]. Molecular markers may aid in the exploration of the variation among the germplasm to select the best candidate parental lines. Similarly, molecular markers may identify heterotic groups or ensure genomic purity of cultivars to achieve heterosis. In addition, molecular markers also assist in backcrossing. Plant breeding conventionally involves several backcrossing steps to enable transfer of one or a few traits to an elite cultivar while retaining most of the recurrent genomes. In general, at least six rounds of backcrossing are required to achieve the desired homozygosity, particularly 
for the selection of traits with low heritability. In contrast, MAS can greatly accelerate this process by utilizing both the flanking markers linked to the trait for selecting the trait and a set of unlinked markers for tracking the recurrent genome. Flanking markers and selection for recombination also reduces "linkage drag," which is the reduction in crop performance due to the cotransfer of undesirable traits that are located in the vicinity of the trait of interest [77]. Typically, a conventional QTL analysis can provide a resolution of approximately $15 \mathrm{cM}$ intervals which may contain hundreds of genes [79]. The availability of a saturated map can potentially reduce this interval to less than $1 \mathrm{cM}$ by backcrossing [78]. Furthermore, MAS enables early selection of traits that are labor and/or cost-intensive to score phenotypically, that are under complex genetic control, or that are manifested late in development. In cases where genotyping by MAS is affordable, this dramatically reduces the number of the plants to be screened in further steps [77, 78].

The major drawbacks of MAS in breeding are high costs of implementation, typically requiring specialized equipment, and the risk of recombination between the marker and the trait that reduces the reliability of MAS to predict phenotype via genotype. The high cost of MAS is particularly relevant in cases where an effective phenotyping method is already established through conventional breeding. Additionally, MAS usually requires the validation of QTLs when applied in different genetic backgrounds. Functional markers, however, may overcome the issue of QTL validation [78]. Despite its drawbacks, MAS has been successfully utilized to improve crops for abiotic stress tolerance, including drought [80], salinity [81], and waterlogging [82] given that the genetic element responsible for the high tolerance is accurately defined and delineated.

Another use of molecular markers is Map-Based Cloning (MBC) where the gene or a QTL linked to a desired trait is isolated via a "mini" physical map. Such a local physical map flanking the gene must be saturated with molecular markers for efficient $\mathrm{MBC}$ [6]. Prior to the construction of highdensity physical maps, MBC approaches were inefficient, particularly due to the difficulty of finding unique probes in repetitive sequences for chromosome walking. Importantly, repeat contents of barley and wheat genomes, two staple crops, are estimated to exceed $80 \%$ of the whole genome [83, 84], potentiating the utility of physical maps. Accordingly, the physical map of chromosome $3 \mathrm{~B}$ provided sufficient data to enable fine mapping of 16 genes and QTLs in chromosome $3 \mathrm{~B}$, none of which had been previously cloned [64].

\section{Comparative Genomics}

For species with largely unexplored genomes, comparative genomics is a promising tool to gain information by utilizing the conservation between closely related plant species. In fact, plant genomes share extensive similarities even between distantly related species (Figure 2, [85]). Among the plant kingdom, grasses have been the focus of comparative genomics analyses due to their high agronomic importance. The extent of genome conservation first became evident by
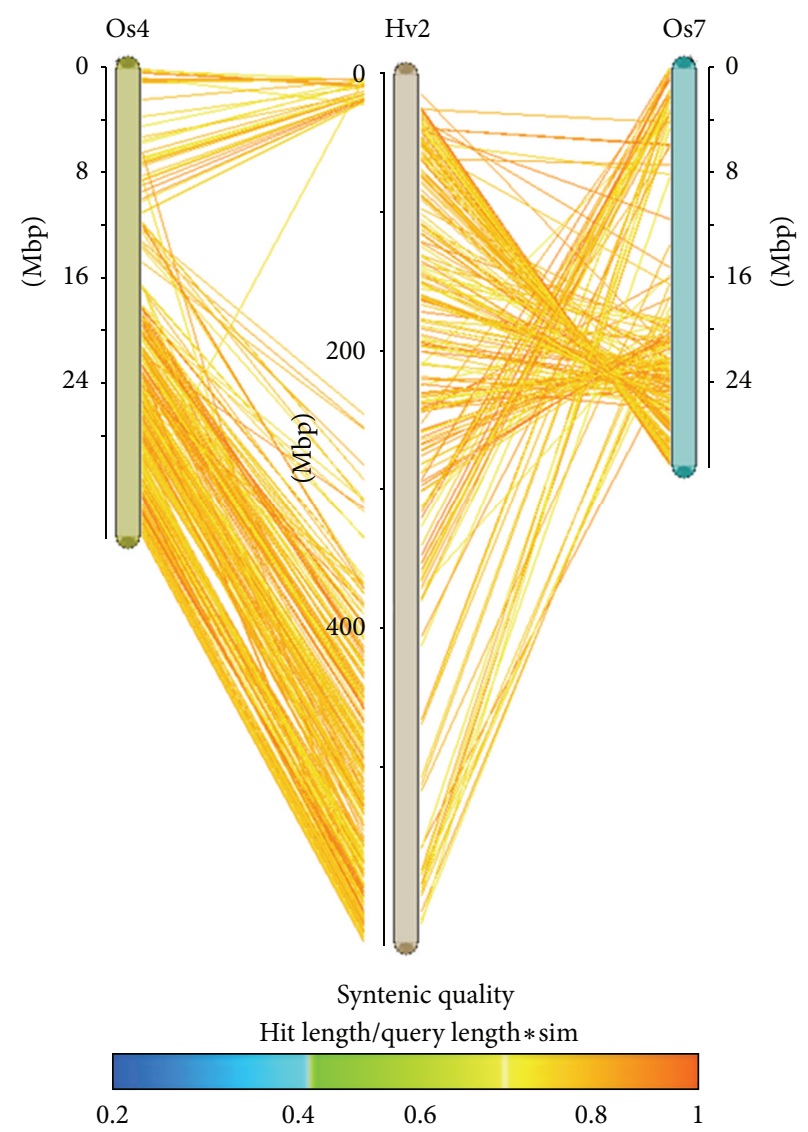

FIgURE 2: Example of colinearity between grass genomes. Analysis of conserved gene sequences between barley (H. vulgare) and rice (O. sativa) shows that many genes are found in colinear (syntenic) order. In this example, chromosome 2 of barley (Hv2, centre) is compared with chromosomes 4 and 7 of rice (Os4 and Os7). Each colored line represents a gene conserved between the two chromosomes, with the color indicating the strength of the syntenic relationship. It is clear that many genes from both ends of Hv2 are colinear with the ends of Os4, while the centre of the chromosome is largely colinear with Os7, but in the reverse order. Image was generated using the CrowsNest Comparative Map Viewer at MIPS (http://mips.helmholtz-muenchen.de/plant/genomes.jsp).

comparative genome mapping studies, which suggested a colinear order of genes and markers shared by genomes of different species. It is noteworthy that plant genomes differ by several orders of magnitude in size; yet these differences generally correspond to intergenic regions. Although detailed analyses have revealed notable rearrangements such as inversions, deletions, and translocations at the molecular level, large-scale colinearity across grass genomes has been exploited for gene discovery and isolation [86-88].

Comparative genomics has contributed significantly to the emergence of the "genome zipper" concept, which enables the determination of a virtual gene order in a partially sequenced genome. Genome zippers compare the fully sequenced and annotated genomes of Brachypodium, sorghum, and rice with various sources of data from less well-studied species, such as genomic survey sequences and 
genetically mapped markers, to predict the gene order and organization in these species $[65,89]$. These genome zippers indicate evolutionary relationships and medium-scale rearrangements, and for the Triticeae provide the closest approximation to a reference genome sequence currently available [65]. However, its reliance on synteny means that recently evolved genes and small-scale rearrangements cannot be explored by this approach.

In addition to syntenic genes that are found in colinear blocks of conserved genes, nonsyntenic genes that are found outside their syntenic location in other genomes also provide valuable insight into genome evolution and speciation. Intriguingly, a recent study focused on the nonconserved portion of wheat and barley genome that suggested novel mechanisms, besides transposable element-driven expansion, have driven the evolution and size of these genomes. Many of these non-syntenic genes exhibited pseudogene characteristics, which may have implications for gene content estimate of these large genomes based on survey sequences [90].

Despite the utility of comparative genomics and genome zippers, it is evident that species-specific genomic features can only be accessed through a fully annotated reference genome sequences. Homoeologous genes with different orthologous relationships are examples of such speciesspecific features [91]. Species-specific rearrangements are also implicated in the formation of gene islands, containing mainly non-syntenic genes, in large crop genomes [92]. Through comparative genomics, Mayer et al. [65] concluded that genomic models can represent the barley genome to a limited extent. Thus, it can be argued that for maximal exploitation of crop genomes, such as wheat and barley, the construction of reference genome sequences scaffolded by highly-saturated physical and genetic maps is indispensable. Accordingly, efforts to accomplish this goal are currently underway (International Wheat Genome Sequencing Consortium for wheat; International Barley Sequencing Consortium for barley).

\section{References}

[1] F. Qin, K. Shinozaki, and K. Yamaguchi-Shinozaki, "Achievements and challenges in understanding plant abiotic stress responses and tolerance," Plant and Cell Physiology, vol. 52, no. 9, pp. 1569-1582, 2011.

[2] M. Kantar, S. J. Lucas, and H. Budak, "Drought stress: molecular genetics and genomics approaches," in Advances in Botanical Research, I. Turkan, Ed., pp. 445-493, Elsevier, Burlington, Mass, USA, 2011.

[3] T. R. Sinclair, "Challenges in breeding for yield increase for drought," Trends in Plant Science, vol. 16, no. 6, pp. 289-293, 2011.

[4] D. Bouchez and H. Höfte, "Functional genomics in plants," Plant Physiology, vol. 118, no. 3, pp. 725-732, 1998.

[5] S. Rudd, "Expressed sequence tags: alternative or complement to whole genome sequences?" Trends in Plant Science, vol. 8, no. 7, pp. 321-329, 2003.

[6] R. K. Varshney, D. A. Hoisington, and A. K. Tyagi, "Advances in cereal genomics and applications in crop breeding," Trends in Biotechnology, vol. 24, no. 11, pp. 490-499, 2006.
[7] K. Yamamoto and T. Sasaki, "Large-scale EST sequencing in rice," Plant Molecular Biology, vol. 35, no. 1-2, pp. 135-144, 1997.

[8] N. Z. Ergen and H. Budak, "Sequencing over 13000 expressed sequence tags from six subtractive cDNA libraries of wild and modern wheats following slow drought stress," Plant, Cell and Environment, vol. 32, no. 3, pp. 220-236, 2009.

[9] V. E. Velculescu, L. Zhang, B. Vogelstein, and K. W. Kinzler, "Serial analysis of gene expression," Science, vol. 270, no. 5235, pp. 484-487, 1995.

[10] M. E. Vega-Sánchez, M. Gowda, and G. L. Wang, “Tag-based approaches for deep transcriptome analysis in plants," Plant Science, vol. 173, no. 4, pp. 371-380, 2007.

[11] P. Breyne and M. Zabeau, "Genome-wide expression analysis of plant cell cycle modulated genes," Current Opinion in Plant Biology, vol. 4, no. 2, pp. 136-142, 2001.

[12] H. Matsumura, S. Nirasawa, and R. Terauchi, "Transcript profiling in rice (Oryza sativa L.) seedlings using serial analysis of gene expression (SAGE)," Plant Journal, vol. 20, no. 6, pp. 719-726, 1999.

[13] W. W. Lorenz and J. F. D. Dean, "SAGE profiling and demonstration of differential gene expression along the axial developmental gradient of lignifying xylem in loblolly pine (Pinus taeda)," Tree Physiology, vol. 22, no. 5, pp. 301-310, 2002.

[14] J. G. Gibbings, B. P. Cook, M. R. Dufault et al., "Global transcript analysis of rice leaf and seed using SAGE technology," Plant Biotechnology Journal, vol. 1, no. 4, pp. 271-285, 2003.

[15] J. Y. Lee and D. H. Lee, "Use of serial analysis of gene expression technology to reveal changes in gene expression in Arabidopsis pollen undergoing cold stress," Plant Physiology, vol. 132, no. 2, pp. 517-529, 2003.

[16] H. Matsumura, S. Reich, A. Ito et al., "Gene expression analysis of plant host-pathogen interactions by SuperSAGE”, Proceedings of the National Academy of Sciences of the United States of America, vol. 100, no. 26, pp. 15718-15723, 2003.

[17] K. L. Nielsen, A. L. Høgh, and J. Emmersen, "DeepSAGEdigital transcriptomics with high sensitivity, simple experimental protocol and multiplexing of samples," Nucleic Acids Research, vol. 34, no. 19, article e133, 2006.

[18] S. Brenner, M. Johnson, J. Bridgham et al., "Gene expression analysis by massively parallel signature sequencing (MPSS) on microbead arrays," Nature Biotechnology, vol. 18, no. 6, pp. 630$634,2000$.

[19] J. Reinartz, E. Bruyns, J. Z. Lin et al., "Massively parallel signature sequencing (MPSS) as a tool for in-depth quantitative gene expression profiling in all organisms," Briefings in Functional Genomics and Proteomics, vol. 1, no. 1, pp. 95-104, 2002.

[20] B. C. Meyers, F. F. Souret, C. Lu, and P. J. Green, "Sweating the small stuff: microRNA discovery in plants," Current Opinion in Biotechnology, vol. 17, no. 2, pp. 139-146, 2006.

[21] K. Nobuta, R. C. Venu, C. Lu et al., "An expression atlas of rice mRNAs and small RNAs," Nature Biotechnology, vol. 25, no. 4, pp. 473-477, 2007.

[22] R. Sunkar, V. Chinnusamy, J. Zhu, and J. K. Zhu, "Small RNAs as big players in plant abiotic stress responses and nutrient deprivation," Trends in Plant Science, vol. 12, no. 7, pp. 301-309, 2007.

[23] M. Nakano, K. Nobuta, K. Vemaraju, S. S. Tej, J. W. Skogen, and B. C. Meyers, "Plant MPSS databases: signature-based transcriptional resources for analyses of mRNA and small RNA," Nucleic Acids Research, vol. 34, pp. D731-D735, 2006. 
[24] M. Jain, A. Nijhawan, R. Arora et al., "F-Box proteins in rice. Genome-wide analysis, classification, temporal and spatial gene expression during panicle and seed development, and regulation by light and abiotic stress," Plant Physiology, vol. 143, no. 4, pp. 1467-1483, 2007.

[25] M. Schena, D. Shalon, R. W. Davis, and P. O. Brown, "Quantitative monitoring of gene expression patterns with a complementary DNA microarray," Science, vol. 270, no. 5235, pp. 467-470, 1995.

[26] D. J. Lockhart, H. Dong, M. C. Byrne et al., "Expression monitoring by hybridization to high-density oligonucleotide arrays," Nature Biotechnology, vol. 14, no. 13, pp. 1675-1680, 1996.

[27] W. A. Rensink and C. R. Buell, "Microarray expression profiling resources for plant genomics," Trends in Plant Science, vol. 10, no. 12, pp. 603-609, 2005.

[28] P. Zimmermann, M. Hirsch-Hoffmann, L. Hennig, and W. Gruissem, "GENEVESTIGATOR. Arabidopsis microarray database and analysis toolbox," Plant Physiology, vol. 136, no. 1, pp. 2621-2632, 2004.

[29] D. Wang, Y. Pan, X. Zhao, L. Zhu, B. Fu, and Z. Li, “Genomewide temporal-spatial gene expression profiling of drought responsiveness in rice," BMC Genomics, vol. 12, article 149, 2011.

[30] R. Kumar, A. Mustafiz, K. K. Sahoo et al., "Functional screening of cDNA library from a salt tolerant rice genotype Pokkali identifies mannose-1-phosphate guanyl transferase gene (OsMPG1) as a key member of salinity stress response," Plant Molecular Biology, vol. 79, no. 6, pp. 555-568, 2012.

[31] A. Singh, A. Pandey, V. Baranwal, S. Kapoor, and G. K. Pandey, "Comprehensive expression analysis of rice phospholipase D gene family during abiotic stresses and development," Plant Signaling and Behavior, vol. 7, no. 7, pp. 847-855, 2012.

[32] N. Z. Ergen, J. Thimmapuram, H. J. Bohnert, and H. Budak, "Transcriptome pathways unique to dehydration tolerant relatives of modern wheat," Functional and Integrative Genomics, vol. 9, no. 3, pp. 377-396, 2009.

[33] T. J. Close, S. I. Wanamaker, R. A. Caldo et al., "A new resource for cereal genomics: 22K Barley GeneChip comes of age," Plant Physiology, vol. 134, no. 3, pp. 960-968, 2004.

[34] M. Luo, J. Liu, R. D. Lee, B. T. Scully, and B. Guo, "Monitoring the expression of maize genes in developing Kernels under drought stress using oligo-microarray," Journal of Integrative Plant Biology, vol. 52, no. 12, pp. 1059-1074, 2010.

[35] A. Ranjan, N. Pandey, D. Lakhwani, N. K. Dubey, U. V. Pathre, and S. V. Sawant, "Comparative transcriptomic analysis of roots of contrasting Gossypium herbaceum genotypes revealing adaptation to drought," BMC Genomics, vol. 13, article 680, 2012.

[36] Y. Utsumi, M. Tanaka, T. Morosawa et al., "Transcriptome analysis using a high-density oligomicroarray under drought stress in various genotypes of cassava: an important tropical crop," DNA Research, vol. 19, no. 4, pp. 335-345, 2012.

[37] R. Loukehaich, T. Wang, B. Ouyang et al., "SpUSP, an annexininteracting universal stress protein, enhances drought tolerance in tomato," Journal of Experimental Botany, vol. 63, no. 15, pp. 5593-5606, 2012.

[38] M. K. Deyholos, "Making the most of drought and salinity transcriptomics," Plant, Cell and Environment, vol. 33, no. 4, pp. 648-654, 2010.

[39] E. A. Bray, "Genes commonly regulated by water-deficit stress in Arabidopsis thaliana," Journal of Experimental Botany, vol. 55, no. 407, pp. 2331-2341, 2004.
[40] J. Yazaki, B. D. Gregory, and J. R. Ecker, "Mapping the genome landscape using tiling array technology," Current Opinion in Plant Biology, vol. 10, no. 5, pp. 534-542, 2007.

[41] K. Mochida and K. Shinozaki, "Genomics and bioinformatics resources for crop improvement," Plant and Cell Physiology, vol. 51, no. 4, pp. 497-523, 2010.

[42] G. Zeller, S. R. Henz, C. K. Widmer et al., "Stress-induced changes in the Arabidopsis thaliana transcriptome analyzed using whole-genome tiling arrays," Plant Journal, vol. 58, no. 6, pp. 1068-1082, 2009.

[43] A. Matsui, J. Ishida, T. Morosawa et al., "Arabidopsis tiling array analysis to identify the stress-responsive genes," Methods in Molecular Biology, vol. 639, pp. 141-155, 2010.

[44] W. Verelst, E. Bertolini, S. de Bodt et al., "Molecular and physiological analysis of growth-limiting drought stress in Brachypodium distachyon leaves," Molecular Plant, vol. 6, no. 2, pp. 311-322, 2013.

[45] C. M. McCallum, L. Comai, E. A. Greene, and S. Henikoff, "Targeting induced local lesions IN genomes (TILLING) for plant functional genomics," Plant Physiology, vol. 123, no. 2, pp. 439-442, 2000.

[46] L. Chen, L. Huang, D. Min et al., "Development and characterization of a new TILLING population of common bread wheat (Triticum aestivum L.)," PLoS ONE, vol. 7, no. 7, Article ID e41570, 2012.

[47] Z. Xin, M. Li Wang, N. A. Barkley et al., "Applying genotyping (TILLING) and phenotyping analyses to elucidate gene function in a chemically induced sorghum mutant population," BMC Plant Biology, vol. 8, article 103, 2008.

[48] B. J. Till, S. H. Reynolds, C. Weil et al., "Discovery of induced point mutations in maize genes by TILLING," BMC Plant Biology, vol. 4, article 12, 2004.

[49] D. G. Caldwell, N. McCallum, P. Shaw, G. J. Muehlbauer, D. F. Marshall, and R. Waugh, "A structured mutant population for forward and reverse genetics in Barley (Hordeum vulgare L.)," Plant Journal, vol. 40, no. 1, pp. 143-150, 2004.

[50] J. L. Cooper, B. J. Till, R. G. Lapor et al., "TILLING to detect induced mutations in soybean," BMC Plant Biology, vol. 8, article 9, 2008.

[51] J. L. Cooper, S. Henikoff, L. Comai, and B. J. Till, "TILLING and ecotilling for rice," Methods in Molecular Biology, vol. 956, pp. 39-56, 2013.

[52] L. de Lorenzo, F. Merchan, P. Laporte et al., "A novel plant leucine-rich repeat receptor kinase regulates the response of Medicago truncatula roots to salt stress," Plant Cell, vol. 21, no. 2, pp. 668-680, 2009.

[53] L. Comai, K. Young, B. J. Till et al., "Efficient discovery of DNA polymorphisms in natural populations by Ecotilling," Plant Journal, vol. 37, no. 5, pp. 778-786, 2004.

[54] S. Negrão, M. C. Almadanim, I. S. Pires et al., "New allelic variants found in key rice salt-tolerance genes: an association study," Plant Biotechnology Journal, vol. 11, no. 1, pp. 87-100, 2013.

[55] S. Yu, F. Liao, F. Wang et al., "Identification of rice transcription factors associated with drought tolerance using the Ecotilling method," PLoS ONE, vol. 7, no. 2, Article ID e30765, 2012.

[56] J. R. Andersen and T. Lübberstedt, "Functional markers in plants," Trends in Plant Science, vol. 8, no. 11, pp. 554-560, 2003.

[57] R. K. Varshney, A. Graner, and M. E. Sorrells, "Genomicsassisted breeding for crop improvement," Trends in Plant Science, vol. 10, no. 12, pp. 621-630, 2005. 
[58] M. Bagge, X. Xia, and T. Lubberstedt, "Functional markers in wheat," Current Opinion in Plant Biology, vol. 10, no. 2, pp. 211216, 2007.

[59] B. Garg, C. Lata, and M. Prasad, "A study of the role of gene TaMYB2 and an associated SNP in dehydration tolerance in common wheat," Molecular Biology Reports, vol. 39, no. 12, pp. 10865-10871, 2012.

[60] R. K. Varshney, S. N. Nayak, G. D. May, and S. A. Jackson, "Nextgeneration sequencing technologies and their implications for crop genetics and breeding," Trends in Biotechnology, vol. 27, no. 9, pp. 522-530, 2009.

[61] P. L. Morrell, E. S. Buckler, and J. Ross-Ibarra, "Crop genomics: advances and applications," Nature Reviews Genetics, vol. 13, no. 2, pp. 85-96, 2011.

[62] C. Feuillet, N. Stein, L. Rossini et al., "Integrating cereal genomics to support innovation in the Triticeae," Functional and Integrative Genomics, vol. 12, no. 4, pp. 573-583, 2012.

[63] E. Paux, P. Sourdille, J. Salse et al., "A physical map of the 1gigabase bread wheat chromosome 3B," Science, vol. 322, no. 5898, pp. 101-104, 2008.

[64] E. Paux, D. Roger, E. Badaeva et al., "Characterizing the composition and evolution of homoeologous genomes in hexaploid wheat through BAC-end sequencing on chromosome 3B," Plant Journal, vol. 48, no. 3, pp. 463-474, 2006.

[65] K. F. X. Mayer, M. Martis, P. E. Hedley et al., "Unlocking the barley genome by chromosomal and comparative genomics," Plant Cell, vol. 23, no. 4, pp. 1249-1263, 2011.

[66] N. Vitulo, A. Albiero, C. Forcato et al., "First survey of the wheat chromosome $5 \mathrm{~A}$ composition through a next generation sequencing approach," PLoS ONE, vol. 6, no. 10, Article ID e26421, 2011.

[67] S. Fluch, D. Kopecky, K. Burg et al., "Sequence composition and gene content of the short arm of rye (Secale cereale) chromosome 1," PLoS ONE, vol. 7, no. 2, Article ID e30784, 2012.

[68] P. Hernandez, M. Martis, G. Dorado et al., "Next-generation sequencing and syntenic integration of flow-sorted arms of wheat chromosome $4 \mathrm{~A}$ exposes the chromosome structure and gene content," Plant Journal, vol. 69, no. 3, pp. 377-386, 2012.

[69] S. J. Lucas, H. Šimková, J. Šafář et al., "Functional features of a single chromosome arm in wheat (1AL) determined from its structure," Functional and Integrative Genomics, vol. 12, no. 1, pp. 173-182, 2012.

[70] H. Takagi, A. Abe, K. Yoshida et al., "QTL-seq: rapid mapping of quantitative trait loci in rice by whole genome resequencing of DNA from two bulked populations," Plant Journal, vol. 74, no. 1, pp. 174-183, 2013.

[71] D. Edwards and J. Batley, "Plant genome sequencing: applications for crop improvement," Plant Biotechnology Journal, vol. 8, no. 1, pp. 2-9, 2010.

[72] J. Mammadov, R. Aggarwal, R. Buyyarapu, and S. Kumpatla, "SNP markers and their impact on plant breeding," International Journal of Plant Genomics, vol. 2012, Article ID 728398, 11 pages, 2012.

[73] D. F. Simola and J. Kim, "Sniper: improved SNP discovery by multiply mapping deep sequenced reads," Genome Biology, vol. 12, no. 6, article 55, 2011.

[74] T. Yoshida, H. Nishida, J. Zhu et al., "Vrn-D4 is a vernalization gene located on the centromeric region of chromosome 5D in hexaploid wheat," Theoretical and Applied Genetics, vol. 120, no. 3, pp. 543-552, 2010.
[75] E. Paux, S. Faure, F. Choulet et al., "Insertion site-based polymorphism markers open new perspectives for genome saturation and marker-assisted selection in wheat," Plant Biotechnology Journal, vol. 8, no. 2, pp. 196-210, 2010.

[76] M. Mohan, S. Nair, A. Bhagwat et al., "Genome mapping, molecular markers and marker-assisted selection in crop plants," Molecular Breeding, vol. 3, no. 2, pp. 87-103, 1997.

[77] B. C. Y. Collard and D. J. Mackill, "Marker-assisted selection: an approach for precision plant breeding in the twenty-first century," Philosophical Transactions of the Royal Society B, vol. 363, no. 1491, pp. 557-572, 2008.

[78] G. O. Edmeades, G. S. McMaster, J. W. White, and H. Campos, "Genomics and the physiologist: bridging the gap between genes and crop response," Field Crops Research, vol. 90, no. 1, pp. 5-18, 2004.

[79] R. Tuberosa, S. Salvi, M. C. Sanguineti, P. Landi, M. Maccaferri, and S. Conti, "Mapping QTLS regulating morpho-physiological traits and yield: case studies, shortcomings and perspectives in drought-stressed maize," Annals of Botany, vol. 89, pp. 941-963, 2002.

[80] M. Ashraf, "Inducing drought tolerance in plants: recent advances," Biotechnology Advances, vol. 28, no. 1, pp. 169-183, 2010.

[81] T. Yamaguchi and E. Blumwald, "Developing salt-tolerant crop plants: challenges and opportunities," Trends in Plant Science, vol. 10, no. 12, pp. 615-620, 2005.

[82] F. Ahmed, M. Y. Rafii, M. R. Ismail et al., "Waterlogging tolerance of crops: breeding, mechanism of tolerance, molecular approaches, and future prospects," BioMed Research International, vol. 2013, Article ID 963525, 10 pages, 2013.

[83] T. Wicker, S. Taudien, A. Houben et al., "A whole-genome snapshot of 454 sequences exposes the composition of the barley genome and provides evidence for parallel evolution of genome size in wheat and barley," Plant Journal, vol. 59, no. 5, pp. 712-722, 2009.

[84] F. Choulet, T. Wicker, C. Rustenholz et al., "Megabase level sequencing reveals contrasted organization and evolution patterns of the wheat gene and transposable element spaces," Plant Cell, vol. 22, no. 6, pp. 1686-1701, 2010.

[85] R. Guyot, F. Lefebvre-Pautigny, C. Tranchant-Dubreuil et al., "Ancestral synteny shared between distantly-related plant species from the asterid (Coffea canephora and Solanum Sp.) and rosid (Vitis vinifera) clades," BMC Genomics, vol. 13, article 103, 2012.

[86] C. Feuillet and B. Keller, "Comparative genomics in the grass family: molecular characterization of grass genome structure and evolution," Annals of Botany, vol. 89, no. 1, pp. 3-10, 2002.

[87] B. Keller and C. Feuillet, "Colinearity and gene density in grass genomes," Trends in Plant Science, vol. 5, no. 6, pp. 246-251, 2000.

[88] F. Li, C. Ma, Q. Chen et al., "Comparative mapping reveals similar linkage of functional genes to QTL of yield-related traits between Brassica napus and Oryza sativa," Journal of Genetics, vol. 91, no. 2, pp. 163-170, 2012.

[89] K. F. X. Mayer, S. Taudien, M. Martis et al., "Gene content and virtual gene order of barley chromosome 1H," Plant Physiology, vol. 151, no. 2, pp. 496-505, 2009.

[90] T. Wicker, K. F. X. Mayer, H. Gundlach et al., "Frequent gene movement and pseudogene evolution is common to the large and complex genomes of wheat, barley, and their relatives," Plant Cell, vol. 23, no. 5, pp. 1706-1718, 2011. 
[91] C. Rustenholz, P. E. Hedley, J. Morris et al., "Specific patterns of gene space organisation revealed in wheat by using the combination of barley and wheat genomic resources," BMC Genomics, vol. 11, no. 1, article 714, 2010.

[92] C. Rustenholz, F. Choulet, C. Laugier et al., "A 3,000-loci transcription map of chromosome $3 \mathrm{~B}$ unravels the structural and functional features of gene islands in hexaploid wheat," Plant Physiology, vol. 157, no. 4, pp. 1596-1608, 2011. 

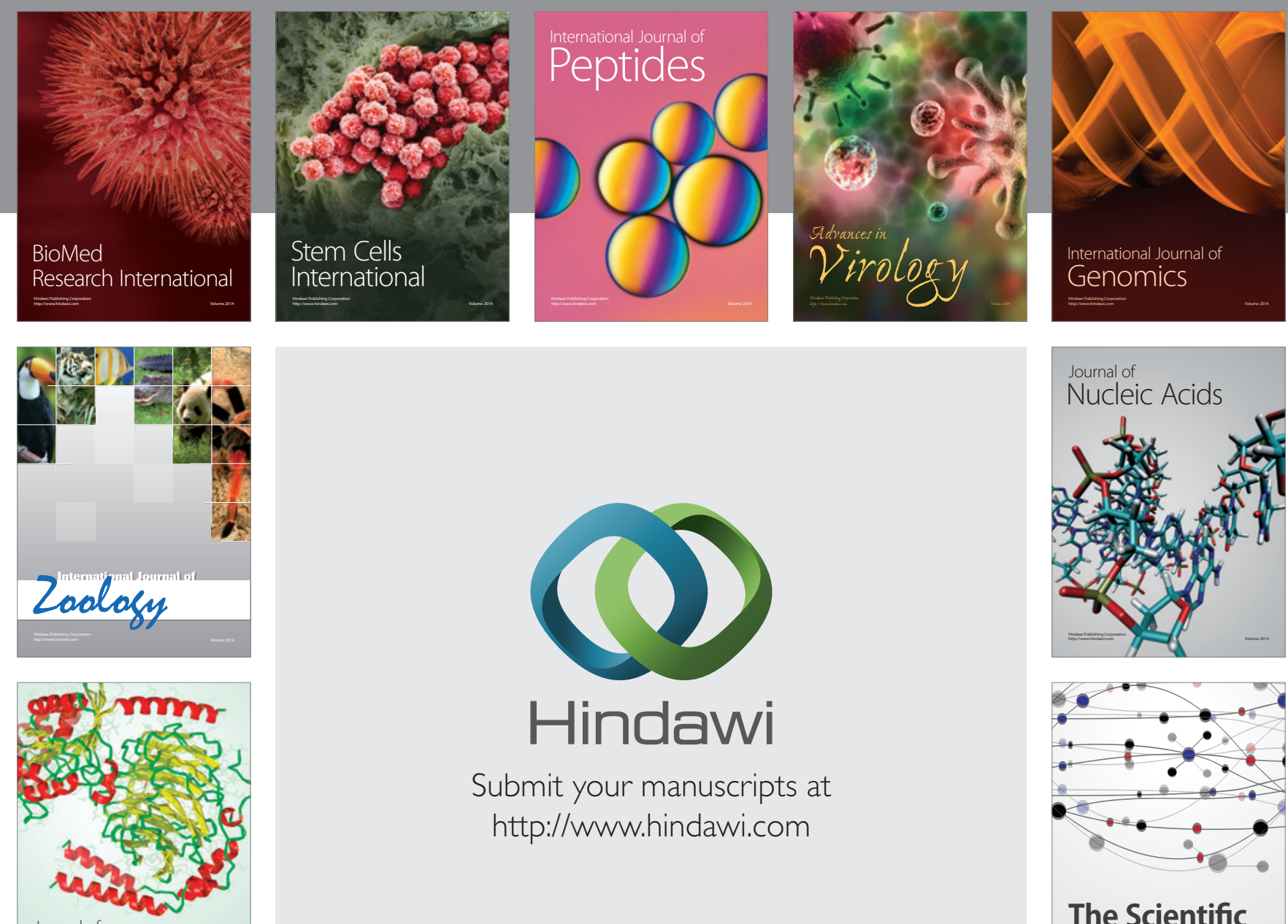

Submit your manuscripts at

http://www.hindawi.com

Journal of
Signal Transduction
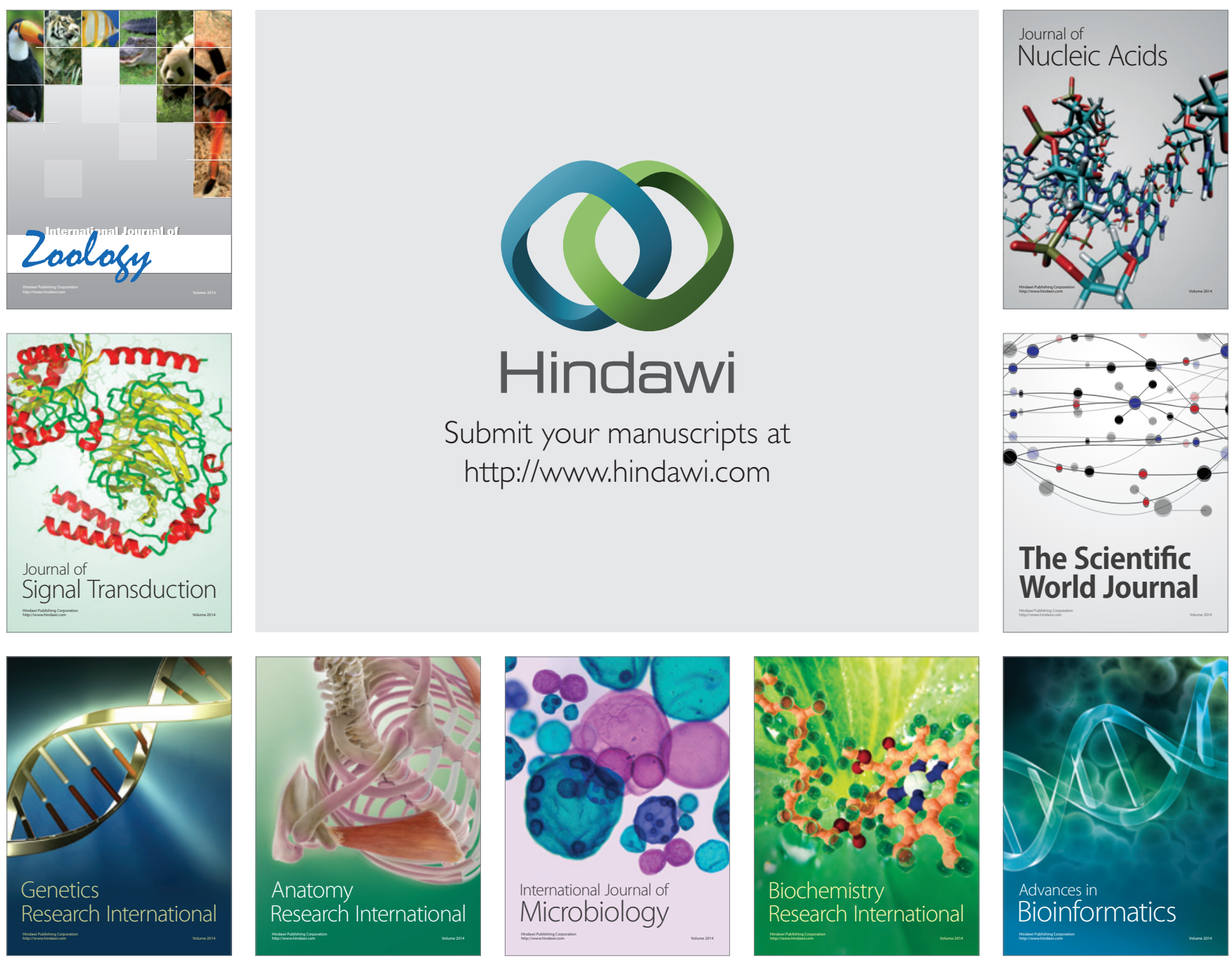

The Scientific World Journal
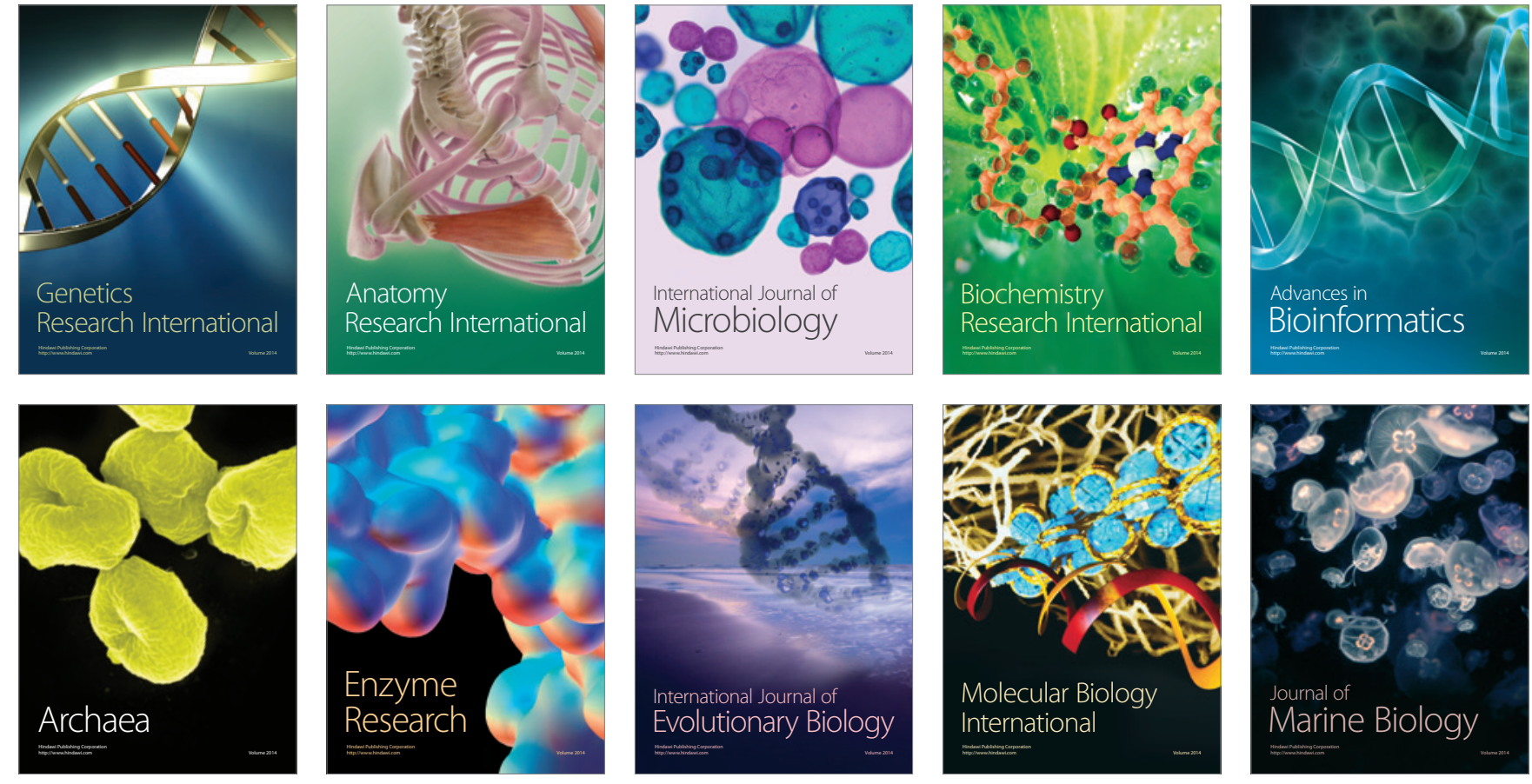